\title{
RNA-binding protein CELF2 inhibits breast cancer cell invasion and angiogenesis by downregulating NFATc1
}

\author{
LIMIN ZHOU ${ }^{1}$ and XIJU XIE ${ }^{2}$ \\ ${ }^{1}$ Department of The Second Section Office of Breast Tumor, Jilin Cancer Hospital, Changchun, \\ Jilin 130000; ${ }^{2}$ Department of Breast Surgery, The First Affiliated Hospital of \\ Nanjing Medical University, Nanjing, Jiangsu 210029, P.R. China
}

Received October 20, 2020; Accepted May 24, 2021

DOI: $10.3892 /$ etm.2021.10330

\begin{abstract}
Breast cancer constitutes a major cause of morbidity and mortality among women in China and worldwide. The aim of the present study was to investigate whether CUGBP Elav-like family member 2 (CELF2) could inhibit breast cancer cell invasion and angiogenesis by downregulating nuclear factor of activated $\mathrm{T}$ cells 1 (NFATc1) expression. The expression of CELF2 and NFATc1 in breast cancer cells and tissues was detected by reverse transcription-quantitative PCR analysis. H\&E staining was used to assess the number of microvessels in tumor tissue. The expression of proteins associated with invasion and angiogenesis and NFATc1 in tumor tissues and transfected cells was examined by western blotting. RNA pull-down assay was used to verify the interaction between CELF2 and NFATc1. Cell proliferation, invasion and tube-forming ability was analyzed using Cell Counting Kit-8, Transwell and HUVEC tube formation assays, respectively. CELF2 expression was found to be decreased in breast cancer cells, whereas CELF2 overexpression suppressed the proliferation and invasion of breast cancer cells and inhibited tumor grow th and angiogenesis. Furthermore, CELF2 overexpression decreased the expression of $\mathrm{N}$-cadherin (N-cad), CD34 and NFATc1 in tumor tissues, whereas NEAFc1 overexpression increased the expression of N-cad and NFATc1 in MCF cells transfected with OverExp-CELF2. CELF2 was found to be inversely associated with NFATc1, and NFATc1 overexpression reversed the effects of CELF2 overexpression. In conclusion, the findings of the present study demonstrated that CELF2 may inhibit breast cancer cell invasion and angiogenesis by downregulating NFATc1.
\end{abstract}

Correspondence to: Dr Xiju Xie, Department of Breast Surgery, The First Affiliated Hospital of Nanjing Medical University, 368 Jiangdong North Road, Nanjing, Jiangsu 210029, P.R. China E-mail: xiju_xie@163.com

Key words: CUGBP Elav-like family member 2, breast cancer, invasion, angiogenesis, nuclear factor of activated $\mathrm{T}$ cells 1

\section{Introduction}

Breast cancer is one of the leading causes of morbidity and mortality among women. The incidence of breast cancer has been increasing annually and it poses a major threat to the survival and quality of life of the patients (1). The three most common types of cancer in women include breast, lung and colorectal cancer, accounting for $50 \%$ of all new cancer diagnoses. Breast cancer alone accounts for $30 \%$ of all cancers in women, and its incidence is rising at a modest rate of $\sim 0.3 \%$ annually (2). In 2015, breast cancer was the most common cancer diagnosed among Chinese women aged 30-59 years, and it was the main cause of cancer-related mortality among women aged $<45$ years in China. Furthermore, the morbidity and mortality rates of breast cancer in China are continuously increasing (3). Therefore, continued research is crucial for identifying effective therapeutic targets for patients with breast cancer.

Nuclear factor of activated T cells (NFAT), a transcription factor found in $\mathrm{T}$ lymphocytes, was originally considered to be a gene regulating T-cell activation (4). The NFAT family consists of multiple members, including NFATc1 (also known as NFAT2), NFATc2 (NFAT1), NFATc3 (NFAT4) and NFATc4 (NFAT3), which are located in the cytoplasm of immune cells in a highly phosphorylated state (5). Once the cell is stimulated, NFATc1 responds to $\mathrm{Ca}^{2+}$-calmodulin signaling by dephosphorylation, and nuclear translocation occurs simultaneously (6). Accumulating evidence has shown that the activation of NFAT signaling does not only promote the occurrence and development of hematological malignancies, but can also accelerate the development of solid tumors. High expression of NFATc1 has been observed in lung, liver and pancreatic cancer $(7,8)$. A recent study also reported that NFATc1 was found to be highly expressed in breast cancer, and that NFATc1 knockdown reduced the proliferation of tumor cells and promoted their apoptosis, which may be due to the effect of the Ras homolog family member A/Rho-associated protein kinase pathway (9).

RNA-binding protein (RBP) is a general term for ubiquitous proteins that can bind to RNA. RBPs bind specifically to RNA and directly or indirectly regulate its function (10). The ENCORI database (http://starbase.sysu.edu.cn/index.php) predicted that CUGBP Elav-like family member 2 (CELF2) is an RBP targeting NFATc1 and that it may exert a regulatory effect on its expression. The expression of CELF2 is low 
in breast cancer due to epigenetic changes, and CELF2 has been shown to possess anticancer properties and inhibit tumor progression (11). However, studies and experimental verification of its relevant mechanisms of action are currently lacking.

The aim of the present study was to investigate the mechanism underlying the anticancer effects of CELF2, and preliminarily evaluate the underlying mechanism by observing the regulatory association between this RBP and the NFAT pathway in breast cancer.

\section{Materials and methods}

Cell culture and transfection. The MCF-10A mammary epithelial cells, the BT-20, T47D, MCF-7 and BT-549 breast cancer cell lines, and HUVECs, were provided by the American Type Culture Collection.

MCF-10A, BT-20, T47D, MCF-7 and BT-549 cells were cultured, digested and passaged in 90\% RPMI-1640 medium supplemented with 10\% FBS (both from Thermo Fisher Scientific, Inc.). MCF-7 cells at the logarithmic growth phase were uniformly inoculated into a 6 -well plate. When the cell confluence had reached $\sim 80 \%$, cells were transfected with empty vector (negative control; NC; $60 \mathrm{nM}$ ), OverExp-CELF2 $(60 \mathrm{nM})$ and OverExp-NFATc1 $(60 \mathrm{nM})$ (all from Shanghai GenePharma Co., Ltd.) using Lipofectamine ${ }^{\circledR} 2000$ (Thermo Fisher Scientific, Inc.) at $37^{\circ} \mathrm{C}$ for $48 \mathrm{~h}$, according to the manufacturer's instructions. Serum-free RPMI-1640 culture medium was used during transfection. Normal culture medium was replaced with RPMI-1640 containing 10\% FBS at $6 \mathrm{~h}$ after transfection, and cells were used for subsequent experiments $48 \mathrm{~h}$ after transfection.

HUVECs were cultured in endothelial cell medium (ECM; ScienCell Research Laboratories, Inc.). When the cell confluence had reached $\sim 80 \%$, HUVECs were transfected with empty vector (NC; $60 \mathrm{nM})$, OverExp-CELF2 (60 nM) and OverExp-NFATc1 $(60 \mathrm{nM})$ using Lipofectamine ${ }^{\circledR} 2000$ (Thermo Fisher Scientific, Inc.) at $37^{\circ} \mathrm{C}$ for $48 \mathrm{~h}$, according to the manufacturer's instructions.

Reverse transcription-quantitative (RT- $q$ ) PCR analysis. A total of $\sim 1 \times 10^{5}$ MCF-7 cells per well were evenly inoculated into 6-well plates and cultured for $24 \mathrm{~h}$. Total RNA was extracted from the cells using TRIzol ${ }^{\circledast}$ reagent (Thermo Fisher Scientific, Inc.) and reverse-transcribed into cDNA using a PrimeScript RT Reagent kit (Takara Biotechnology Co., Ltd.) according to the manufacturer's instructions. Next, a TaqMan Universal PCR Master Mix kit (Thermo Fisher Scientific, Inc.) was used to conduct qPCR to determine the relative expression of CELF2 and NFATc1. The thermocycling conditions were as follows: Initial denaturation at $95^{\circ} \mathrm{C}$ for $30 \mathrm{sec}$, followed by 40 cycles at $95^{\circ} \mathrm{C}$ for $25 \mathrm{sec}, 60^{\circ} \mathrm{C}$ for $35 \mathrm{sec}$ and $72^{\circ} \mathrm{C}$ for $35 \mathrm{sec}$. The following primer pairs were used for the qPCR: CELF2 forward, 5'-CTGGCGGGA AACAAACTCTG-3' and reverse, 5'-TCTAAGCCCTTGGCCTCCTC-3'; NFATc1 forward, 5'-CCACCGAGCCCACTACGAGA-3' and reverse, 5'-CAGGATTCCGGCACAGTCAAT-3'; GAPDH forward, 5'-AAGGTGAAGGTCGGAGTCAAC-3' and reverse, 5'-GGG GTCATTGATGGCAACAATA-3'. mRNA levels were quantified using the $2^{-\Delta \Delta \mathrm{Cq}}$ method and normalized to the internal reference gene GAPDH (12).
Xenograft model. MCF-7 cells cultured for 6 days were collected and a $1 \times 10^{7} / \mathrm{ml}$ cell suspension was created using PBS. A total of 15 female BALB/c nude mice (age, 6-8 weeks; weight, $18-20 \mathrm{~g}$ ) were housed in an environmentally controlled room $\left(22 \pm 2^{\circ} \mathrm{C} ; 12\right.$-h light/dark cycle; $50-65 \%$ humidity) and were given free access to food and water. After being allowed to acclimate for 1 week, each mouse was subcutaneously injected with $1 \times 10^{6}$ cells into the right armpit. Mouse weight and tumor diameter were measured once every 3 days after injection. The maximum tumor size obtained was $<1,000 \mathrm{~mm}^{3}$. The experiment finished after 3 weeks, and the mice were euthanized with intraperitoneal injection of $150 \mathrm{mg} / \mathrm{kg}$ pentobarbital sodium and the tumors were resected. The animal experimental protocols were approved by the Animal Ethics Committee of Jilin Cancer Hospital (approval no. JLCH2020-0052).

$H \& E$ staining. The tumor tissues were fixed in $10 \%$ neutral methylal for $24 \mathrm{~h}$ at room temperature and washed fully with flowing water. Following dehydration with 70, 80, 90, 95 and $100 \%$ ethanol, the tissues were transparentized with xylene, embedded in paraffin, cut into $4-\mu \mathrm{m}$ sections and stained with H\&E for $3 \mathrm{~min}$ at room temperature. The sections were then observed and images were captured using a light microscope (Olympus Corporation) at the magnification of $x 400$.

Western blot analysis. The lysis products were extracted from tumor tissues and transfected cells using RIPA lysis buffer (Beyotime Institute of Biotechnology), and the protein concentration was measured with a BCA protein assay kit. After high-temperature denaturation, the proteins (30 $\mu \mathrm{g} /$ lane) were loaded and separated via 12\% SDS-PAGE and transferred to a PVDF membrane. The membrane was then blocked with 5\% skimmed milk powder for $2 \mathrm{~h}$ at room temperature, and incubated with antibodies against $\mathrm{N}$-cadherin (N-cad; cat. no. ab76011; dilution, 1:5,000; Abcam), CD34 (cat. no. ab81289; dilution, 1:10,000; Abcam), NFATc1 (cat. no. ab124292; dilution, 1:5,000; Abcam) and GAPDH (cat. no. ab8245; dilution, 1:5,000; Abcam) overnight at $4^{\circ} \mathrm{C}$. Subsequently, the membrane was incubated with anti-rabbit HRP-linked IgG secondary antibody (1:1,000; cat. no. 7074) or anti-mouse HRP-linked IgG secondary antibody (1:1,000; cat. no. 7076) (both from Cell Signaling Technology, Inc.) at room temperature for $2 \mathrm{~h}$. ECL solution (MilliporeSigma) was used for color development. Then, protein bands were examined using Image Lab ${ }^{\mathrm{TM}}$ software (cat. no. 1709690; Bio-Rad Laboratories, Inc.) and ImageJ software (v1.8.0.112; National Institutes of Health) was used for semi-quantitative protein analysis.

RNA pull-down assay. Briefly, HUVECs were lysed with $500 \mu 1$ RIPA lysis buffer (Beyotime Institute of Biotechnology) and incubated with biotinylated NFATc1 probes (Shanghai GenePharma Co., Ltd.) at $4^{\circ} \mathrm{C}$ for $2 \mathrm{~h}$. Then, $50 \mu 1$ streptavidin magnetic beads (Thermo Fisher Scientific, Inc.) were added to each sample, which was incubated at $4^{\circ} \mathrm{C}$ for $2 \mathrm{~h}$. The beads were washed with lysis buffer and the binding proteins in the pull-down products were collected for use in the western blot analysis as aforementioned (13). 

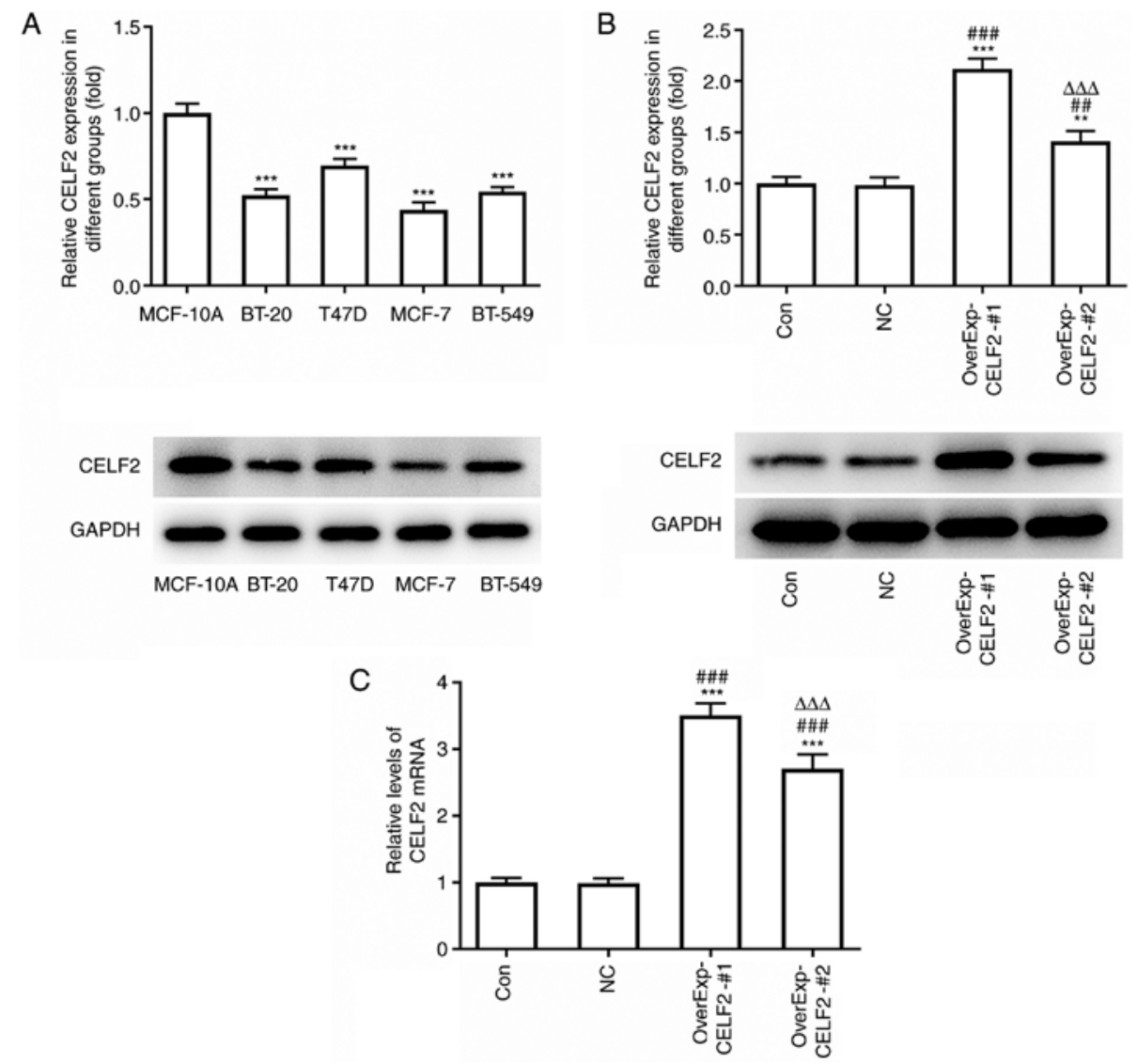

Figure 1. CELF2 expression is decreased in breast cancer cells. (A) Protein expression of CELF2 in mammary epithelial cells and breast cancer cells was detected by western blot analysis. ${ }^{* * *} \mathrm{P}<0.001$ vs. MCF-10A cells. (B) Protein expression of CELF2 in MCF-7 cells transfected with OverExp-CELF2-\#1/2 was examined by western blot analysis. ${ }^{* * *} \mathrm{P}<0.01$ and ${ }^{* * * *} \mathrm{P}<0.001$ vs. Con group. ${ }^{\# \#} \mathrm{P}<0.01$ and ${ }^{\# \# \#} \mathrm{P}<0.001$ vs. NC group. ${ }^{\Delta \Delta \Delta} \mathrm{P}<0.001$ vs. OverExp-CELF2-\#1 group. (C) mRNA expression of CELF2 in MCF-7 cells transfected with OverExp-CELF2-\#1/2 was analyzed by reverse transcription-quantitative PCR analysis. ${ }^{* * *} \mathrm{P}<0.001$ vs. Con group. ${ }^{\# \#} \mathrm{P}<0.001$ vs. $\mathrm{NC}$ group. ${ }^{\Delta \Delta \Delta} \mathrm{P}<0.001$ vs. OverExp-CELF2-\#1 group. CELF2, CUGBP Elav-like family member 2; Con, control; NC, negative control.

Cell Counting Kit(CCK)-8assay. MCF-7 cells (4,000 cells/well) and HUVECs ( $1 \times 10^{5}$ cells/well) were inoculated into a $96-$ well plate and cultured for $24 \mathrm{~h}$ after transfection. Then, $48 \mathrm{~h}$ after culture, $10 \mu \mathrm{l}$ CCK-8 solution (Beyotime Institute of Biotechnology) was added to each well and incubated at $37^{\circ} \mathrm{C}$ for $1 \mathrm{~h}$. The optical density at $450 \mathrm{~nm}$ was determined using a microplate reader (Thermo Fisher Scientific, Inc.).

Transwell assay. The matrix glue was solidified in a cell incubator for $1 \mathrm{~h}$ after diluting with $50 \mu \mathrm{l}$ basic glue in a small chamber. After transfection for $24 \mathrm{~h}, \mathrm{MCF}-7$ cells at the logarithmic growth phase were evenly inoculated into the upper chamber of a 24-well Transwell chamber with RPMI-1640 medium at a density of $5 \times 10^{4}$ cells/well and cultured for $24 \mathrm{~h}$ at $37^{\circ} \mathrm{C}$. The lower chamber contained RPMI-1640 medium with $10 \%$ FBS. The cells in the upper surface of the filter (8- $\mu \mathrm{m}$ pore size) were removed with a cotton swab, and the cells invading to the lower surface of the filter were fixed with $4 \%$ formaldehyde for $30 \mathrm{~min}$ at room temperature and stained with $0.2 \%$ crystal violet solution for $60 \mathrm{~min}$ at room temperature and counted at least six random microscopic fields under a light microscope (Olympus Corporation; magnification, $\mathrm{x} 100)$.

HUVEC tube formation assay. HUVECs (5x10 $/$ well) were added into $300 \mu \mathrm{l}$ ECM and seeded on a 48 -well plate coated with $300 \mu \mathrm{l}$ Matrigel/well. After 2 days of incubation at $37^{\circ} \mathrm{C}$, tube formation in the Matrigel was observed under a phase-contrast microscope (magnification, $\mathrm{x} 4$ ).

Statistical analysis. SPSS 20.0 software (IBM Corp.) was used for analysis, and the quantitative data of normal distribution are presented as the mean \pm SD from three experimental repeats. An unpaired t-test and one-way ANOVA with Tukey's post hoc test were used for comparison between two and multiple groups, respectively. $\mathrm{P}<0.05$ was considered to indicate a statistically significant difference.

\section{Results}

CELF2 expression is decreased in breast cancer cells. The protein expression level of CELF2 was found to be decreased in BT-20, MCF-7 and BT-549 cells, but was not notably different in T47D cells, compared with MCF-10A cells. Moreover, CELF2 expression was the lowest in MCF-7 cells; therefore, MCF-7 cells were selected for the subsequent experiments (Fig. 1A). After MCF-7 cells were transfected with OverExp-CELF2-\#1/2, CELF2 expression markedly increased. It was found that CELF2 protein expression (Fig. 1B) and CELF2 mRNA expression (Fig. 1C) were both higher in the OverExp-CELF2-\#1 group; thus, OverExp-CELF2-\#1 was selected for subsequent experiments. 
A
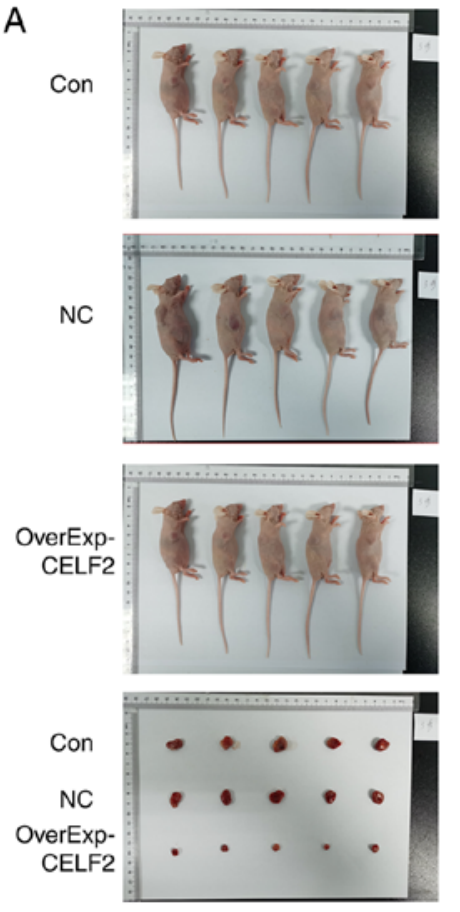

$\mathrm{F}$

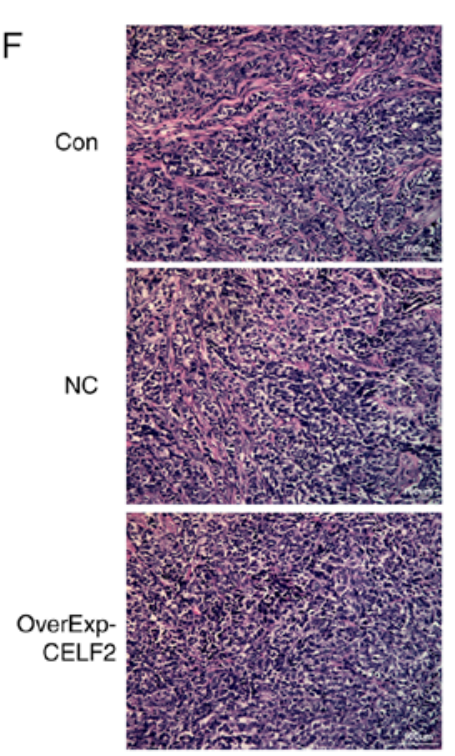

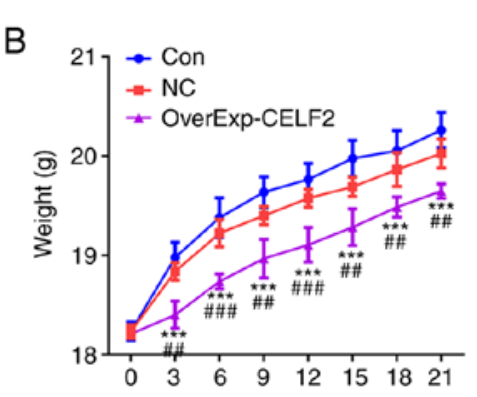

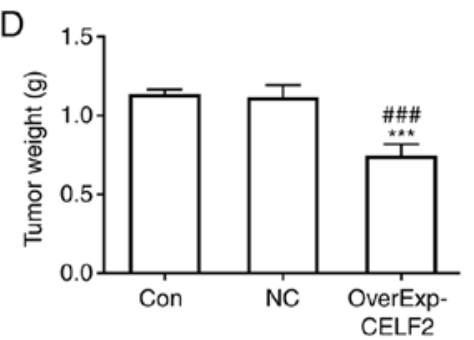

G
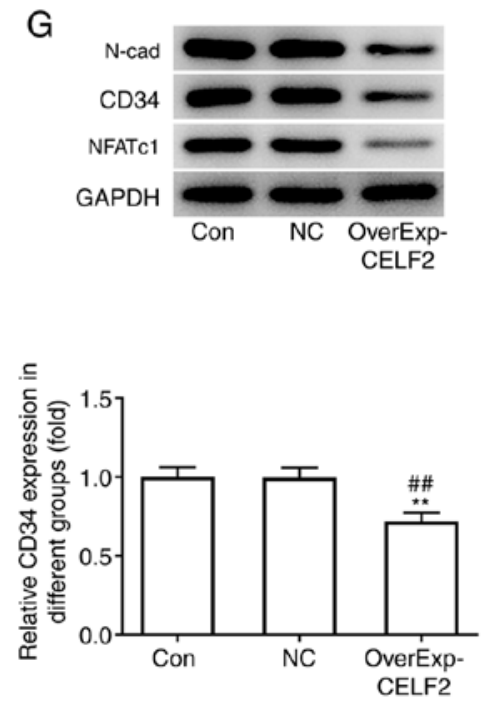

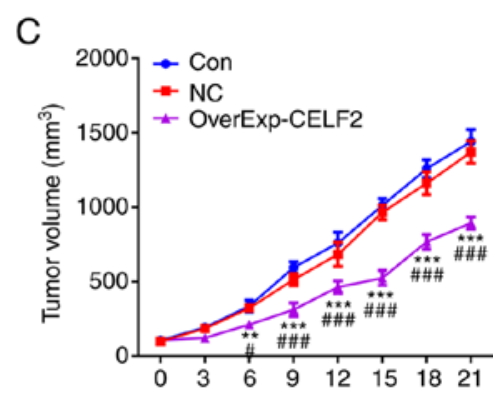

$\mathrm{E}$
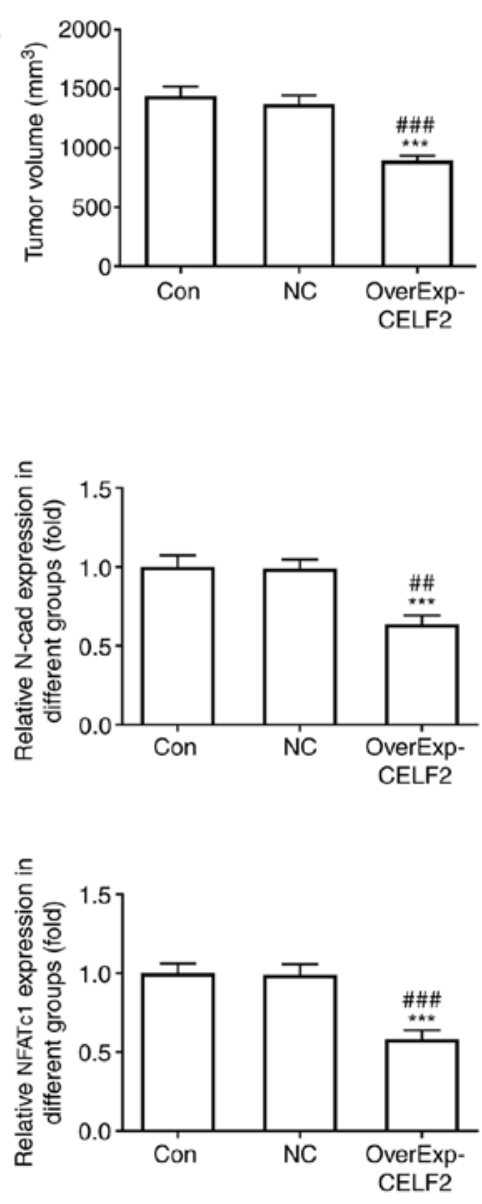

Figure 2. CELF2 overexpression inhibits tumor growth and angiogenesis. (A) Mice and tumor in the three groups. (B) Mouse weight changed from day 0 to day 21. ${ }_{* * * * *} \mathrm{P}<0.001$ vs. Con group. ${ }^{\# \#} \mathrm{P}<0.01$ and ${ }^{\# \# \#} \mathrm{P}<0.001$ vs. $\mathrm{NC}$ group. (C) Tumor volume changed from day 0 to day 21 . ${ }^{* *} \mathrm{P}<0.01$ and ${ }^{* * *} \mathrm{P}<0.001$ vs. Con group. ${ }^{\#} \mathrm{P}<0.05$ and ${ }^{\# \# \#} \mathrm{P}<0.001$ vs. NC group. (D) Tumor weight at day $21 .{ }^{* * *} \mathrm{P}<0.001$ vs. Con group. ${ }^{\# \# \#} \mathrm{P}<0.001$ vs. NC group. (E) Tumor volume at day $21{ }^{*{ }^{* * *}} \mathrm{P}<0.001$ vs. Con group. ${ }^{\# \#} \mathrm{P}<0.001$ vs. NC group. (F) Angiogenesis was analyzed by HUVEC tube formation assay; scale bar, $100 \mu \mathrm{m}$. (G) Western blot analysis was performed to detect the expression of proteins associated with invasion and angiogenesis and NFATc1 in tumor tissues. ${ }^{* *} \mathrm{P}<0.01$ and ${ }^{* * * *} \mathrm{P}<0.001$ vs. Con group. ${ }^{\# \#} \mathrm{P}<0.01$ and

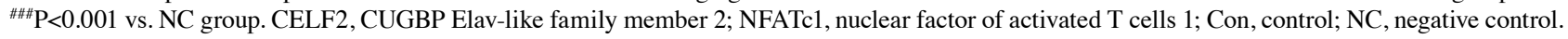

CELF2 overexpression inhibits tumor growth and angiogenesis. Macroscopic images of the mice and tumors in the three groups are presented in Fig. 2A. CELF2 overexpression led to weight loss in the mice (Fig. 2B) and decrease in tumor volume from day 0 to day 21 (Fig. 2C). At the end of the experiment, tumor weight (Fig. 2D) and tumor volume (Fig. 2E) were decreased in the OverExp-CELF2 group. Furthermore, CELF2 overexpression decreased the number of microvessels in tumor tissues (Fig. 2F). The expression levels of N-cad, CD34 (angiogenesis-promoting factor) and NFATc1 were significantly reduced in the OverExp-CELF2 group (Fig. 2G).
CELF2 is combined with NFATc1. The RNA pull-down assay results demonstrated the association between CELF2 and NFATc1 (Fig. 3A). NFATc1 expression was increased in MCF-7 cells transfected with OverExp-NFATc1-\#1/2, and NFATc1 expression was highest in the OverExp-NFATc1-\#1 group. Therefore, OverExp-NFATc1-\#1 was selected for the subsequent experiments (Fig. 3B).

CELF2 overexpression suppresses the viability and invasion of breast cancer cells by downregulating NFATc1. CELF2 overexpression decreased the viability (Fig. 4A) and invasion ability (Fig. 4B and C) of MCF-7 cells, and these effects were reversed 
A

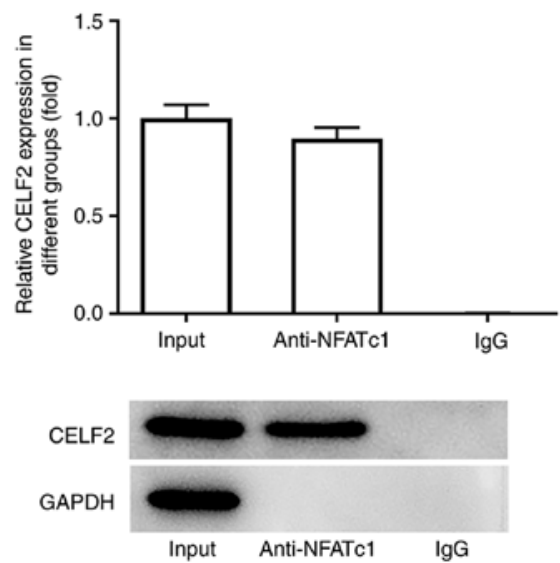

B

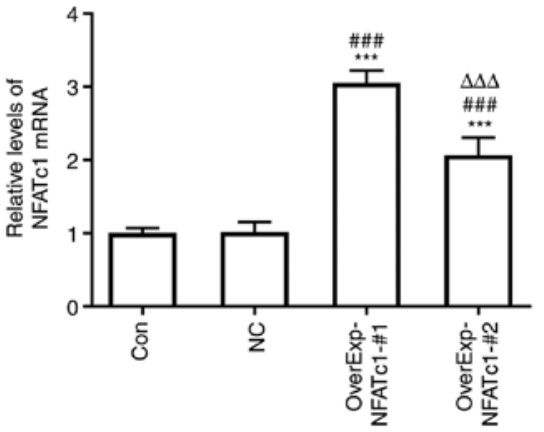

Figure 3. Association of CELF2 with NFATc1. (A) The association of CELF2 and NFATc1 was confirmed by RNA pull-down assay. (B) mRNA expression of NFATc1 in MCF-7 cells transfected with OverExp-NFATc1-\#1/2 was examined by reverse transcription-quantitative PCR analysis. ${ }^{* * *} \mathrm{P}<0.001$ vs. Con group. ${ }^{\# \#} \mathrm{P}<0.001$ vs. $\mathrm{NC}$ group. ${ }^{\Delta \Delta \Delta} \mathrm{P}<0.001$ vs. OverExp-NFATc1-\#1 group. CELF2, CUGBP Elav-like family member 2; NFATc1, nuclear factor of activated T cells 1; Con, control; NC, negative control.
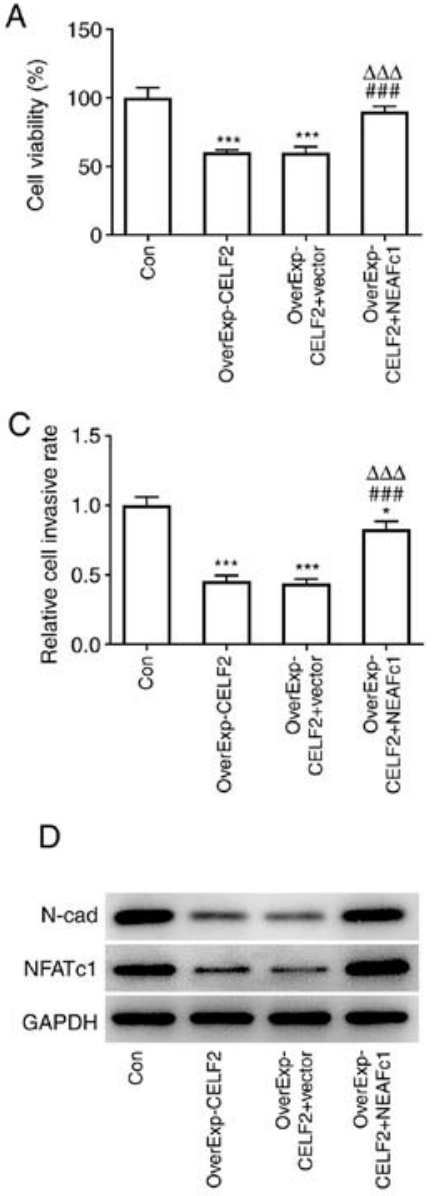

B

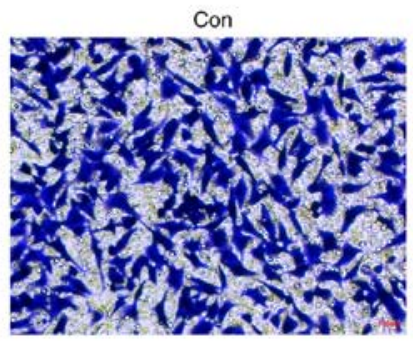

OverExp-CELF2+vector

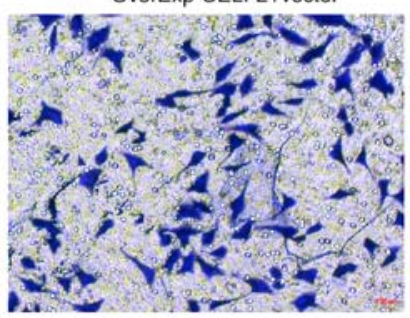

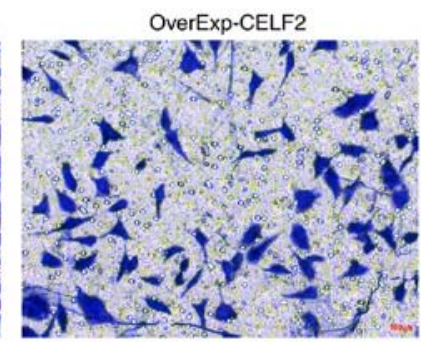

OverEXPCELF2+NEAFC1

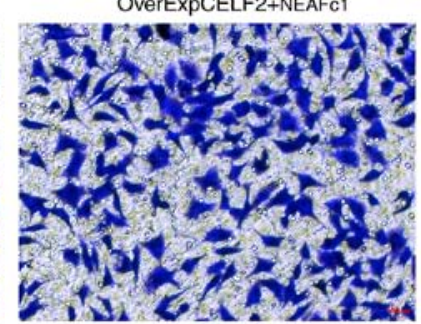

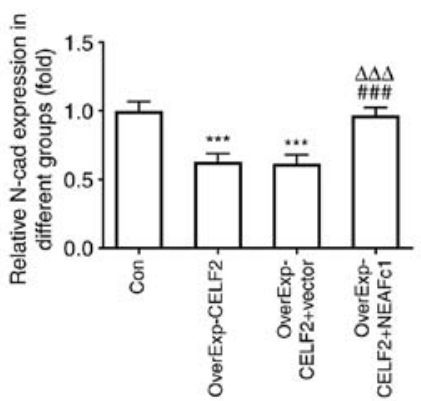

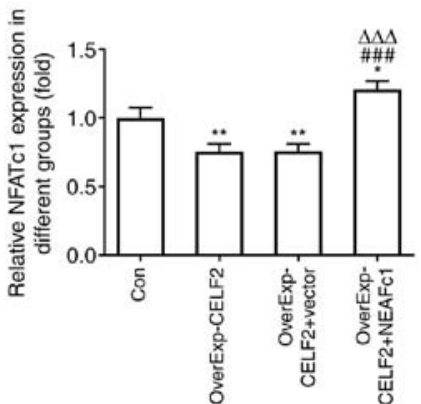

Figure 4. CELF2 overexpression suppresses viability and invasion of breast cancer cells by downregulating NFATc1. (A) Cell Counting Kit-8 assay was used to examine the viability of MCF-7 cells transfected with OverExp-CELF2 and OverExp-NFATc1. ${ }^{* * *} \mathrm{P}<0.001$ vs. Con group. ${ }^{* \# \# P} \mathrm{P}<0.001$ vs. OverExp-CELF2 group. ${ }^{\Delta \Delta} \mathrm{P}<0.001$ vs. OverExp-CELF2 + vector group. (B and C) Transwell assay was used to examine the invasion of MCF-7 cells transfected with OverExp-CELF2 and OverExp-NFATc1; scale bar, $100 \mu \mathrm{m}$. ${ }^{*} \mathrm{P}<0.05$ and ${ }^{* * * *} \mathrm{P}<0.001$ vs. Con group. ${ }^{\# \# \#} \mathrm{P}<0.001$ vs. OverExp-CELF2 group. ${ }^{\Delta \Delta \Delta} \mathrm{P}<0.001$ vs. OverExp-CELF2 + vector group. (D) Western blot analysis was used to examine the expression of proteins associated with invasion and NFATc1 in transfected cells. "P<0.05, ${ }^{* *} \mathrm{P}<0.01$ and ${ }^{* * * *} \mathrm{P}<0.001$ vs. Con group. ${ }^{\# \# \#} \mathrm{P}<0.001$ vs. OverExp-CELF2 group. ${ }^{\Delta \triangle} \Delta \mathrm{P}<0.001$ vs. OverExp-CELF2 + vector group. CELF2, CUGBP Elav-like family member 2; NFATc1, nuclear factor of activated T cells 1; Con, control; NC, negative control.

by NEAFc1 overexpression. Moreover, CELF2 overexpression decreased the expression levels of N-cad and NFATc1, which was reversed by NEAFc1 overexpression (Fig. 4D).
CELF2 overexpression suppresses HUVEC tube formation by downregulating NFATc1. CELF2 overexpression suppressed the viability and angiogenesis of HUVECs, and NEAFc1 


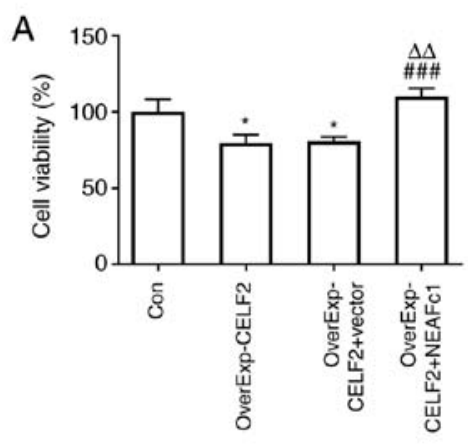

B
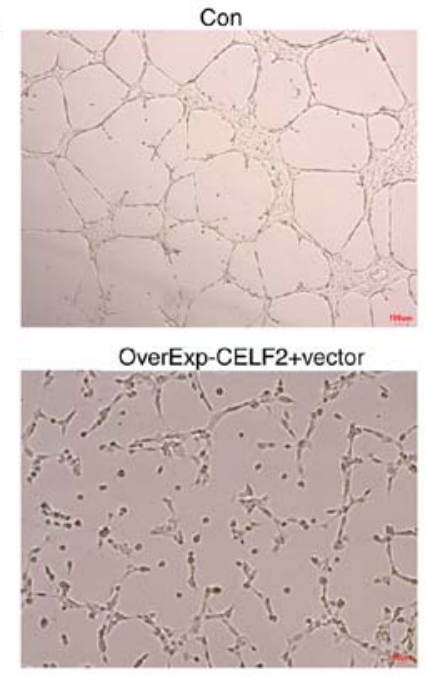

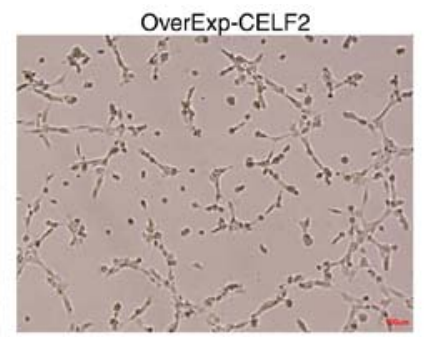

OverExPCELF2+NEAFc1

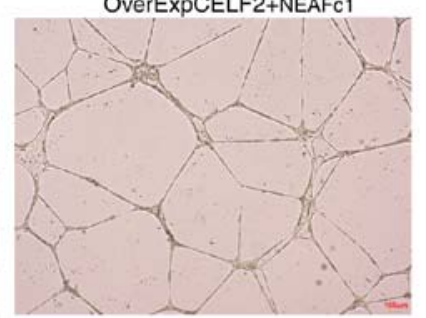

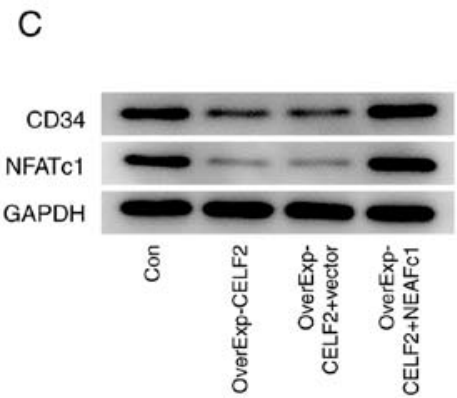
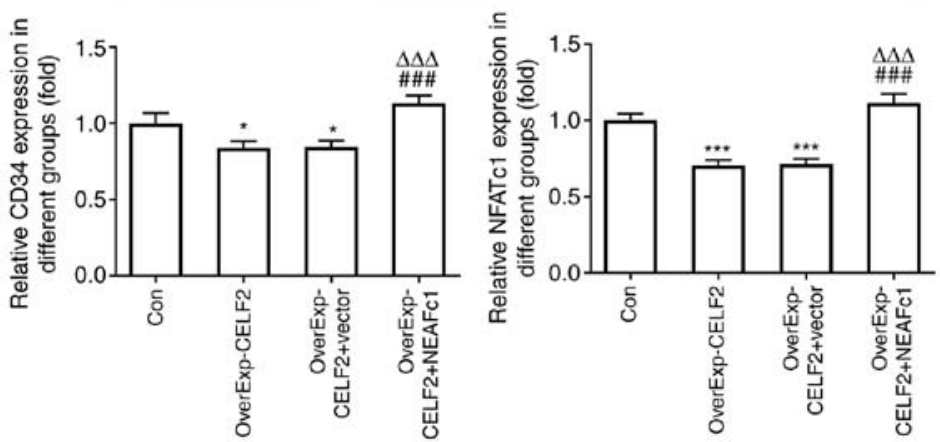

Figure 5. CELF2 overexpression suppresses HUVEC tube formation by downregulating NFATc1. (A) Cell Counting Kit-8 assay was used to examine the viability of HUVECs transfected with OverExp-CELF2 and OverExp-NFATc1. ${ }^{*} \mathrm{P}<0.05$ vs. Con group. ${ }^{\# \#} \mathrm{P}<0.001$ vs. OverExp-CELF2 group. ${ }^{\Delta \Delta} \mathrm{P}<0.01$ vs. OverExp-CELF2 + vector group. (B) HUVEC tube formation assay was used to examine the angiogenic ability of HUVECs transfected with OverExp-CELF2 and OverExp-NFATc1; scale bar, $100 \mu \mathrm{m}$. (C) Western blot analysis was used to examine the expression of proteins associated with angiogenesis and NFATc1 in transfected cells. ${ }^{*} \mathrm{P}<0.05$ and ${ }^{* * *} \mathrm{P}<0.001$ vs. Con group. ${ }^{\# \# \#} \mathrm{P}<0.001$ vs. OverExp-CELF2 group. ${ }^{\Delta \Delta} \mathrm{P}<0.001$ vs. OverExp-CELF2 + vector group. CELF2, CUGBP Elav-like family member 2; NFATc1, nuclear factor of activated T cells 1; Con, control; NC, negative control.

overexpression reversed the effects of CELF2 overexpression (Fig. 5A and B). It was also found that the expression levels of CD34 and NFATc1 in HUVECs were suppressed by CELF2 overexpression, which was reversed by NEAFc1 overexpression (Fig. 5C).

\section{Discussion}

Breast cancer is a heterogeneous disease and is one of the leading causes of mortality among women worldwide (14). It has been reported that there are 1.67 million new cases of breast cancer annually, accounting for $11.88 \%$ of all cancer cases (15). Although a variety of risk factors, such as family history, exogenous hormones, obesity and reproductive factors, have been identified for breast cancer, early cancer detection and further improvement of treatment outcomes remain significant challenges for clinicians (16-18).

The RBP CELF2 was initially identified in colchicine-induced neuroblastoma cells (19). CELF2 expression is downregulated in colon tumor tissues and may be a potential tumor suppressor protein (20), and restoration of CELF2 expression inhibits the proliferation of breast cancer cells (11). MicroRNA (miR)-615-3p, which promotes gastric cancer cell proliferation and migration by downregulating CELF2 expression, has been found to be highly expressed, while CELF2 is underexpressed, in gastric cancer cells and tumor tissues (21). It has also been reported that CELF2 expression is decreased in non-small cell lung carcinoma (NSCLC) tissues, and that CELF2 overexpression suppresses both Akt phosphorylation and cell proliferation in NSCLC (22). In the present study, it was observed that CELF2 expression was decreased in MCF-7 cells, and CELF2 overexpression suppressed the viability and invasion of MCF-7 cells, as well as tumor growth and angiogenesis.

As a transcription factor, NFATc1 serves an important role in tumors. Overexpression of NFATC1 promotes the proliferation of ovarian cancer cells and the occurrence of tumors by regulating the ERK1/2/P38 MAPK signaling pathway (23). Furthermore, activation of NFATc1 may upregulate cyclooxygenase-2 expression, thus promoting the invasion of human glioma cells (24). It has also been demonstrated that inhibition of NFATc1 may suppress the proliferative, migratory and invasive abilities of prostate cancer cells, possibly by decreasing the expression of c-myc and pyruvate kinase isozymes M1/M2 (25). NFATc1 is highly expressed in human serous/mucinous ovarian cancer and downregulation of NFATc1 suppresses cell cycle progression, invasion and migration, and promotes the apoptosis of ovarian cancer cells (26). In the present study, after MCF-7 cells were co-transfected with OverExp-CELF2 and OverExp-NFATc1, it was observed that NFATc1 overexpression weakened the effect of CELF2 overexpression and 
promoted the viability and invasion of MCF-7 cells. In addition, after HUVECs were co-transfected with OverExp-CELF2 and OverExp-NFATc1, it was demonstrated that NFATc1 overexpression weakened the effect of CELF2 overexpression and promoted the viability and angiogenesis of HUVECs.

In conclusion, the results of the present revealed that CELF2 expression is downregulated in breast cancer cells and that CELF2 may inhibit breast cancer cell invasion and angiogenesis by downregulating the expression of NFATc1. Moreover, NFATc1 overexpression may partially reverse the effects of CELF2 overexpression on breast cancer cells.

\section{Acknowledgements}

Not applicable.

\section{Funding}

No funding was received.

\section{Availability of data and materials}

The datasets used and/or analyzed during the current study are available from the corresponding author on reasonable request.

\section{Authors' contributions}

XX conceived the study and revised the manuscript. LZ performed the experiments, data analysis, organized the figures and was responsible for the draft manuscript. LZ and $\mathrm{XX}$ confirm the authenticity of all the raw data. Both authors have read and approved the final manuscript.

\section{Ethics approval and consent to participate}

The animal experimental protocols were approved by the Animal Ethics Committee of Jilin Cancer Hospital (approval no. JLCH2020-0052).

\section{Patient consent for publication}

Not applicable.

\section{Competing interests}

The authors declare that they have no competing interests.

\section{References}

1. Harbeck N and Gnant M: Breast cancer. Lancet 389: 1134-1150, 2017.

2. Siegel RL, Miller KD and Jemal A: Cancer statistics, 2020. CA Cancer J Clin 70: 7-30, 2020.

3. Chen W, Zheng R, Baade P, Zhang S, Zeng H, Bray F, Jemal A, $\mathrm{Yu}$ X and He J: Cancer statistics in China, 2015. CA Cancer J Clin 66: 115-132, 2016.

4. Irnaten M, Zhdanov A, Brennan D, Crotty T, Clark A, Papkovsky D and O'Brien C: Activation of the NFAT-calcium signaling pathway in human lamina cribrosa cells in glaucoma. Invest Ophthalmol Vis Sci 59: 831-842, 2018.

5. Lu WC, Xie H, Tie XX, Wang R, Wu AH and Shan FP: NFAT-1 hyper-activation by methionine enkephalin (MENK) significantly induces cell apoptosis of rats C6 glioma in vivo and in vitro. Int Immunopharmacol 56: 1-8, 2018.
6. He RL, Wu ZJ, Liu XR, Gui LX, Wang RX and Lin MJ: Calcineurin/NFAT signaling modulates pulmonary artery smooth muscle cell proliferation, migration and apoptosis in monocrotaline-induced pulmonary arterial hypertension rats. Cell Physiol Biochem 49: 172-189, 2018.

7. Müller MR and Rao A: NFAT, immunity and cancer: A transcription factor comes of age. Nat Rev Immunol 10: 645-656, 2010.

8. Ouyang Z, Guo X, Chen X, Liu B, Zhang Q, Yin Z, Zhai Z, Qu X, Liu X, Peng D, et al: Hypericin targets osteoclast and prevents breast cancer-induced bone metastasis via NFATc1 signaling pathway. Oncotarget 9: 1868-1884, 2018.

9. Liu M, Wei L, Wang Y,Liu P,Liu X and Zhang M: Effects of NFATc1 on proliferation and apoptosis of breast cancer cells by regulating RhoA /ROCK signaling pathway. Mod Oncol 28: 562-568, 2020.

10. He $\mathrm{Y}$ and Ding $\mathrm{M}$ : Research progress on the interaction between RNA binding proteins and RNA-proteins. Sci Technol Innovation: 10-13, 2020 .

11. Piqué L, Martinez de Paz A, Piñeyro D, Martínez-Cardús A Castro de Moura M, Llinàs-Arias P, Setien F, Gomez-Miragaya J, Gonzalez-Suarez E, Sigurdsson S, et al: Epigenetic inactivation of the splicing RNA-binding protein CELF2 in human breast cancer. Oncogene 38: 7106-7112, 2019.

12. Livak KJ and Schmittgen TD: Analysis of relative gene expression data using real-time quantitative PCR and the 2(-Delta Delta C(T)) method. Methods 25: 402-408, 2001.

13. Dou YQ, Kong P, Li CL, Sun HX, Li WW, Yu Y, Nie L, Zhao LL, Miao SB, Li XK, et al: Smooth muscle SIRT1 reprograms endothelial cells to suppress angiogenesis after ischemia. Theranostics 10: 1197-1212, 2020.

14. Jemal A, Bray F, Center M, Ferlay J, Ward E and Forman D: Global cancer statistics. CA Cancer J Clin 61: 69-90, 2011.

15. Ferlay J, Soerjomataram I, Dikshit R, Eser S, Rebelo M, Parkin D, Forman D and Bray F: Cancer incidence and mortality worldwide: Sources, methods and major patterns in GLOBOCAN 2012. Int J Cancer 136: E359-E386, 2014.

16. Snietura M and Lange D: Current ASCO/CAP 2013 recommendations for testing for HER2 status in breast cancer. Pol J Pathol 65 (Suppl 2): S32-S41, 2014 (In Polish).

17. Naja F, Fadel RA, Alameddine M, Aridi Y, Zarif A, Hariri D, Mugharbel A, Khalil M, Nahleh Z and Tfayli A: Complementary and alternative medicine use and its association with quality of life among Lebanese breast cancer patients: A cross-sectional study. BMC Complement Altern Med 15: 444, 2015.

18. Węsierska-GądekJ and Mauritz M: Why (multi)targeting of cyclin-dependent kinases is a promising therapeutic option for hormone-positive breast cancer and beyond. Future Med Chem 8: 55-72, 2016.

19. Li D, Bachinski LL and Roberts R: Genomic organization and isoform-specific tissue expression of human NAPOR (CUGBP2) as a candidate gene for familial arrhythmogenic right ventricular dysplasia. Genomics 74: 396-401, 2001

20. Ramalingam S, Ramamoorthy $P$, Subramaniam D and Anant S: Reduced expression of RNA binding protein CELF2, a putative tumor suppressor gene in colon cancer. Immunogastroenterology 1: 27-33, 2012.

21. Wang J, Liu L, Sun Y, Xue Y, Qu J, Pan S, Li H, Qu H, Wang J and Zhang $\mathrm{J}$ : miR-615-3p promotes proliferation and migration and inhibits apoptosis through its potential target CELF2 in gastric cancer. Biomed Pharmacother 101: 406-413, 2018.

22. Yeung YT, Fan S, Lu B, Yin S, Yang S, Nie W, Wang M, Zhou L, Li T, Li X, et al: CELF2 suppresses non-small cell lung carcinoma growth by inhibiting the PREX2-PTEN interaction. Carcinogenesis 41: 377-389, 2020.

23. Xu W, Gu J, Ren Q, Shi Y, Xia Q, Wang J, Wang S, Wang Y and Wang J: NFATC1 promotes cell growth and tumorigenesis in ovarian cancer up-regulating c-Myc through ERK1/2/p38 MAPK signal pathway. Tumour Biol 37: 4493-4500, 2016.

24. Wang L, Wang Z, Li J, Zhang W, Ren F and Yue W: NFATc1 activation promotes the invasion of $\mathrm{U} 251$ human glioblastoma multiforme cells through COX-2. Int J Mol Med 35: 1333-1340, 2015.

25. Liu Y, Liang T, Qiu X, Ye X, Li Z, Tian B and Yan D: Down-regulation of Nfatcl suppresses proliferation, migration, invasion, and warburg effect in prostate cancer cells. Med Sci Monit 25: 1572-1581, 2019.

26. Li L, Duan Z, Yu J and Dang HX: NFATc1 regulates cell proliferation, migration, and invasion of ovarian cancer SKOV3 cells in vitro and in vivo. Oncol Rep 36: 918-928, 2016.

This work is licensed under a Creative Commons Attribution-NonCommercial-NoDerivatives 4.0 International (CC BY-NC-ND 4.0) License. 